\title{
Synchronous Bilateral Multiple Typical Pulmonary Carcinoid Tumors: A Unique Case with 10 Typical Carcinoids
}

\author{
Alkın Yazıcıoğlu, Erdal Yekeler, Pınar Bıcakcıoğlu, Esra Özaydın', Nurettin Karaoğlanoğlu \\ Department of Thoracic Surgery, Atatürk Chest Disease and Thoracic Surgery Training and Research Hospital, Ankara, Turkey \\ 1Department of Pathology, Atatürk Chest Disease Training and Research Hospital, Ankara, Turkey
}

\section{ABSTRACT}

Bilateral multiple typical carcinoid tumors of the lung are uncommon malignancies. We discuss the case of a 64 year-old female with a nonproductive cough as the initial symptom. Thoracic computed tomography revealed multiple nodular lesions on both sides, which were initially misdiagnosed as multiple metastases of the lung with an unknown primary. After resection of nodules in the right hemithorax, pathologic examination revealed a typical carcinoid tumor. Bilateral sequential thoracotomy was performed and all ten nodules, (six on the right side and four on the left side), were treated by sublobar resection due to poor respiratory function. Pathological examination revealed all nodules to be typical carcinoid tumors. Following the resections, the patient has remained disease-free for ten months.

Key Words: Pulmonary carcinoid, typical carcinoid, bilateral, synchronous

\section{Introduction}

Bronchial carcinoids are uncommon malignant neoplasms, believed to derive from specialized neuroendocrine cells known as Kulchitsky cells (1). They account for $1-2 \%$ of all lung neoplasms and, of all carcinoid tumors, approximately $25 \%$ are located in the respiratory tract (1-3). Typical carcinoid tumors may be cured after surgical resection and a 10-year patient survival rate of $87 \%$ has been reported (2). We report on a patient with bilateral multiple pulmonary lesions treated by sequential wedge resections.

\section{Case Report}

A 64 year-old female patient was first admitted to a state hospital with the symptom of a nonproductive cough. A chest $\mathrm{x}$-ray revealed bilateral multiple pulmonary nodules and she was subsequently referred to our hospital. The patient was initially admitted with a diagnosis of lung metastases of unknown origin. Her medical history was positive for hypertension treated with calcium channel blockers; and chronic depression treated with escitalopram. No tobacco or alcohol addiction was present. There were no abnormal findings on physical examination.

A computed tomography (CT) scan of the chest revealed bilateral multiple nodules (Figure 1a, b). Laboratory investigations were normal. Respiratory function tests showed poor respiratory function with forced expiratory volume in one second $\left(\mathrm{FEV}_{1}\right)$ of only $0.65 \mathrm{~L}$ (39\% of predicted value). A positron emission tomography (PET) scan was performed preop- eratively and calculated low metabolic activity in the nodules with no primary focus and unknown tumor origin.

With the initial diagnosis of multiple lung metastases of unknown origin, the patient underwent a right thoracotomy for pathological examination. Wedge resection was performed of the largest nodule located in the upper lobe and another nodule from the lower lobe. Subsequent frozen examination revealed a typical carcinoid in both nodules. There were four more nodules on the right side and all nodules were excised with mediastinal lymph node dissection. Pathologic examination indicated all six nodules as typical carcinoid tumors without lymph node metastases (Figure 2).

Three weeks later a left thoracotomy with wedge resection was performed of four pulmonary nodules, one nodule upper lobe and three lower lobe, with mediastinal lymphadenectomy. On pathologic examination, all nodules were also determined as typical carcinoid tumors and no lymph node metastases were detected. Therefore, the patient had a total of 10 typical carcinoid nodules resected (six on the right side and four on the left side). After 10 months, the patient's progress was uneventful with disease-free survival.

\section{Discussion}

Pulmonary carcinoids are uncommon malignant tumors and account for $1-2 \%$ of all pulmonary malignancies (1). Of all pulmonary carcinoids, $80-85 \%$ display typical histology (4). The presentation of bronchial carcinoids in both hemithorax is rarer $(1,3)$. Those tumors may be initially misdiagnosed as metastatic cancer with an unknown primary $(2,3,5)$. In a re- 
view of the English language medical literature, there were only 6 case reports describing bilateral synchronous typical pulmonary carcinoids, of which only 3 cases were bilateral multiple synchronous tumors.

Akashiba et al. (5) described a case who had diffuse bilateral multiple nodular lesions in both hemithorax, initially believed to be metastatic cancer with an unknown primary site. Ten years after this case, Camargo et al. (1) reported a second case of bilateral multiple pulmonary carcinoids with two nodules on the left side and only one on the right. Yazici et al. (3) described a similar case with bilateral and multiple synchronous typical pulmonary carcinoids, two tumors on each hemithorax. Therefore, our case, with 10 nodules in total, was the fourth report of bilateral synchronous multiple typical carcinoids of the lung.

Carcinoids are classically located as single nodules close to the hilus; they may, rarely, present as peripheral and, exceptionally, as multifocal (2). In our case, many nodules were seen on CT images, distributed throughout almost every lobe and segment of both lungs. Consequently, the patient was initially diagnosed with lung metastases of unknown origin.

Positron emission tomography scans generally demonstrate low-level uptake of fluorodeoxyglucose in carcinoid tumors. Patients with carcinoid tumors are more likely to have a negative PET scan than those with other types of lung tumors (4). Our patient also had similar PET findings, with a maximal standard uptake value of only 1.22 . Although this value suggested that a malignant origin was unlikely, it could not exclude a tumor or metastases.

The treatment of choice for carcinoid tumors is complete surgical resection (5). The goal of resection is complete excision of the tumor while sparing as much lung tissue as possible. Carcinoids located in the periphery of the lung are often suitable for limited resection, such as large wedge resection or segmentectomy (4). However, according to Camargo et al. (1), formal lobectomy with lymph node dissection remains the standard procedure for surgical management of peripheral carcinoids. Nonanatomical resections are exposed to a high risk of recurrence, according to Yazici et al. (3).
Patients with typical carcinoid tumors have an excellent prognosis, with a 10 year survival rate of up to $87 \%$ after complete anatomical resection including mediastinal lymph node dissection (2). Anatomical resection was not performed in our case because the nodules were bilateral and multiple and the patient had insufficient respiratory function. The nodules were therefore removed by wedge resection, and a bilateral lymphadenectomy was performed.

The study by Beshay et al. (2) proposed a CT-scan every year for 5 years and an annual chest x-ray thereafter, to exclude development of metachronous carcinoid tumors. We are planning to follow this procedure in the follow-up of our patient.

\section{Conclusion}

Although multiple lung nodules seen on radiological investigations are mainly caused by metastases, synchronous bilateral multiple carcinoids of the lung should be kept on mind, especially in the absence of an unknown primary localization.

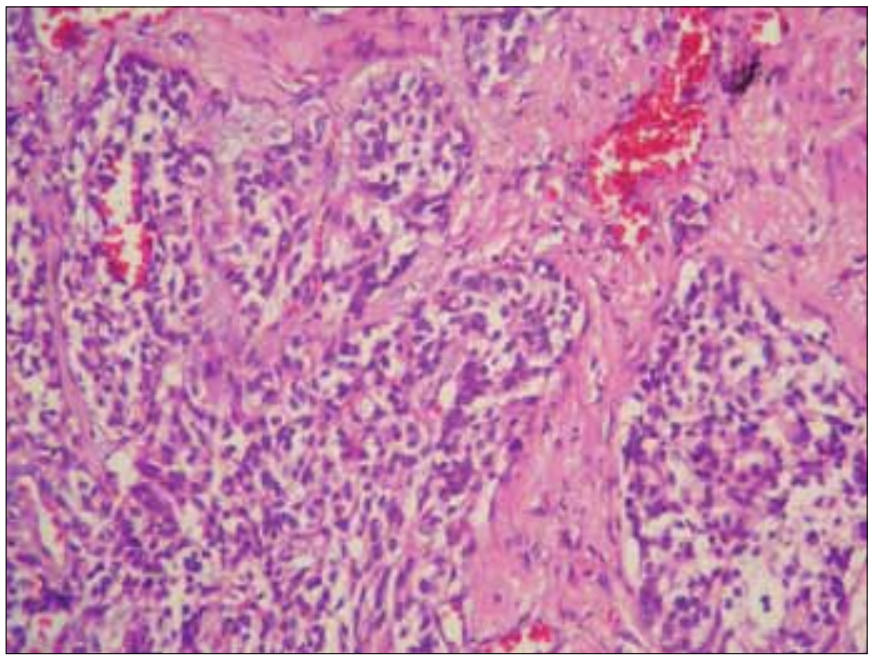

Figure 2. Cell groups of eosinophilic granular cytoplasm and vascular structures with monotone appearance

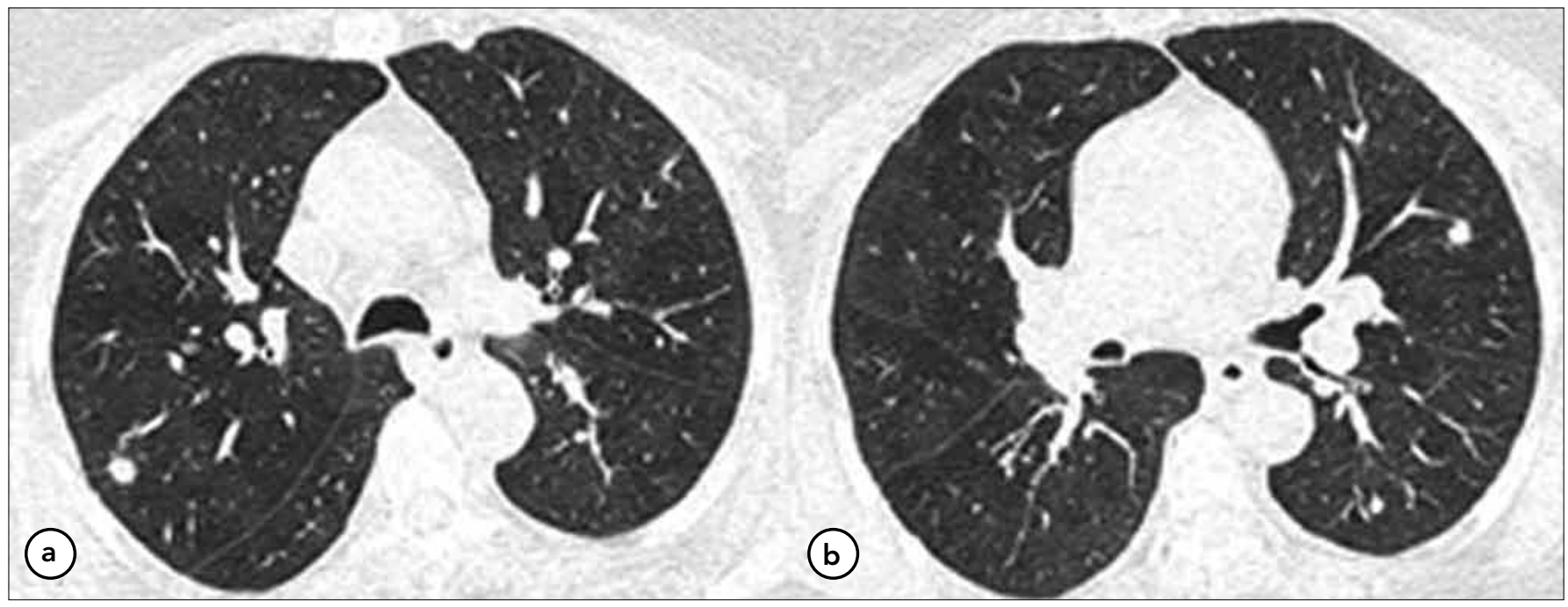

Figure 1. $(a, b)$ Thorax CT scan revealed multiple nodules on both hemithorax 


\section{Conflict of Interest}

No conflict of interest was declared by the authors.

\section{References}

1. Camargo SM, Machuca TN, Moreira AL, Schio SM, Moreira JS, Camargo JJ. Multiple synchronous bronchial carcinoid tumors: report of a case. Thorac Cardiovasc Surg 2009;57:58-60. [CrossRef]

2. Beshay M, Roth $T$, Stein R, Schmid RA. Synchronous bilateral typical pulmonary carcinoid tumors. Eur J Cardiothorac Surg 2003;23:251-3. [CrossRef]
3. Yazici U, Gulhan E, Agackiran Y, Tastepe I, Yaran P. Synchronous bilateral multiple typical pulmonary carcinoid tumors. Ann Thorac Surg 2010;89:1278-80. [CrossRef]

4. Patterson A, Cooper JD, Deslauriers J, Lerut AEMR, Luketich JD, Rice TW. Pearson's Thoracic and Esophageal Surgery. Third edition Philadelphia 2008.p.699-703.

5. Akashiba T, Matsumoto K, Kosaka N, Saito O, Horie T, Nemoto N. Multifocal peripheral bronchial carcinoid tumor. Respirol 1999;4:199-201. [CrossRef] 